\title{
Comparison of stress separation procedures. experiments versus theoretical formulation
}

\author{
Jorge Guillermo Díaz Rodríguez ${ }^{*}$
}

${ }^{a}$ Escuela de ingeniería mecánica. Universidad Industrial de Santander. Carrera 27, calle 9. Bucaramanga, Colombia

\begin{tabular}{l}
\hline A R T I C L E I N F O \\
\hline Article history: \\
Received 20 September 2021 \\
Accepted 26 January 2022 \\
Available online \\
29 January 2022 \\
\hline Keywords: \\
Stress separation \\
Keyhole sample \\
DIC \\
TSA \\
Photo-elasticity \\
Stress analysis \\
Airy stress function
\end{tabular}
\begin{abstract}
A B S T R A C T
The article deals with stress separation using different experimental techniques and their comparison to numerical and theoretical solutions. The method is applied to a keyhole sample coupon, to which measurements of fringe order, temperature or displacement were made using photo-elasticity, TSA (Thermo-elastic Stress Analysis) and DIC (Digital Image Correlation). The results are compared to FEM simulations (Finite Element Method) using the stress concentration factor $\left(K_{t}\right)$ as a benchmark. Additionally, an Airy stress function is proposed and tested against obtained measurements. The comparison of $K_{t}$ shows agreement among measurements as well as numerical and theoretical results. It is concluded that the presented method can be used for isotropic materials subjected to the plane stress, where stress variation through-thickness is negligible.
\end{abstract}

(C) 2022 Growing Science Ltd. All rights reserved.

\section{Introduction}

In experimental mechanics, optical methods, such as Thermo-elastic Stress Analysis (TSA) or photo-elasticity, do not provide independent measurements of either strain or displacement fields (Marsavina \& Tomlinson, 2013; Solarguen et al. 2009; Tabanyukhova, 2019). Measured stresses obtained with these whole field techniques show biaxial components as one value that has to be decomposed (Freire \& Voloshin, 2009; Sakagami, et al., 2004). So useful information can be extracted and properly analyzed. Several methods have been proposed to decompose photo-elasticity stress fields. Anthony et al. (2016) as well as Yoneyama et. al. (2005) derived methods combining photo-elasticity and interferometry to do so. Allison (1998) proposed taking advantage of the symmetry in a stress field to separate a biaxial stress state. However, this method would be limited to a symmetrical stress state such as pure loading modes in a cracked sample (Molteno \& Becker, 2015), which was similar to what was done by Tabanyukhov (2019) but applied only to tangential stresses with manually counted fringe order. Sagakami et al. (2004), Dulie-Smith (1995), and Murakami \& Yoshimura (1995) have independently proposed methods to perform stress separation obtained with at least two whole-field techniques. Moreover, Solarguen et al., (2009) and Ramesh (2000) have summarized methods to perform stress separation based on photo-elasticity measurements. In all cases to decompose the measured stresses, it is required a second technique measurement, or calibration against a known stress value, usually done within the experiment.

On the other hand, stress functions are mathematical equations that describe a biaxial state of stress for a particular load/geometry combination and were widely used until the popularization of the Finite Element Method (FEM). Niedenfuhr (1957) showed recommendations on how to write a bi-harmonic equation stress function but only using polynomic functions. Selvadurai (2000) made similar recommendations but with a broader type of equations. Rao and Nomura (2006) proposed functions for circular inclusions in solid media. More recently, Cavaco et al. (2018) used polynomial functions for stress * Corresponding author.

E-mail addresses: jgdiazro@correo.uis.edu.co (J. G. D. Rodríguez) 
analysis in pipes due to soil-pipe interaction and Jobin et. al. (2020) proposed one function to fit measured displacement fields in a cracked epoxy composite. Finally, there are rules of thumb on how to select a stress function, mainly based on Michell's general solution (Sadd, 2014).

Biaxial stresses are present under two or more orthogonal loads or a sharp change in geometry. Kirsch in 1898 was the first one who successfully tackled and deducted a stress solution around an infinite plate with a circular hole. In 1913, Inglis expanded the solution to elliptical holes showing that stress will grow as the ellipse flattens to the point it will show a singularity (Sadd, 2014). Brewster described photo-elasticity in 1815 but, it was not until 1930 that works of Coker and Filon in Europe and Frocht in the US described a detailed experimental framework for photo-elasticity (Freire \& Voloshin, 2009; Masuda, 2004) which was when biaxial stress field were observed. Their work laid the grounds for optically measured fields as opposed to widely used singular point measurements, such as strain gauges (SG) or the more recent Fiber Bragg Grating (FBG) sensors (Triana-Infante et al. 2014). Although less used nowadays, photo-elasticity is a low cost technique, easy to implement and with well-known analysis and procedures (Masuda, 2004; Vishay, 2011). Alternatively, the modern and widely used technique of Digital Image Correlation (DIC) gives orthogonal displacement measurements (Castillo et al. 2020) but also, it gives errors when measuring displacement fields close to sharp edges or close to out-of-plane geometries due to the error associated with lower correlation coefficients found when there is a discontinuity (Sutton et al. 2009; Vormwald et al. 2017). On the other hand, one of the Thermo Elastics Stress Analysis (TSA) main advantages in fracture mechanics is that the stress intensity factor can be obtained directly by calculating the cyclic stress in front of the crack propagation direction. Extensive applications of TSA take place in civil, maritime, oil and gas, petrochemical and aerospace industries. It is a powerful tool to validate FEM analysis (Marsavina \& Tomlinson, 2013).

In this research to evaluate a method to separate stress, an experiment was performed on a sample which could be tested with different experimental techniques. The same test coupon was tested at elastic loads and measurements taken with photoelasticity, and TSA. To validate the calibration of experimental measures, a Finite Element Analysis (FEM) was performed and, results from a third experimental measure, Digital Image Correlation (DIC) were taken. The paper describes the use of these experimental techniques to measure stress and the combination of some of them to estimate the stress concentration factor in a keyhole sample. Furthermore, this paper tests a method to separate stresses that are measured using independent techniques and compares them with a FEM simulation and a proposed stress function.

\section{Liteature review}

This section describes briefly the techniques used, and some schemes used to perform stress separation.

\subsection{TSA}

The surface temperature of a body is a function of the volumetric heat generation, and the emission of energy at the surface. Thereby, when a body is subjected to tensile stress, its temperature is slightly reduced. Conversely, when subjected to a compressive stress, a slight temperature increase is produced. When the body is subjected to cyclic loading, one can measure the small change in surface temperature $(\Delta \mathrm{T})$, which can be in the order of $1 * 10^{-3} \mathrm{~K}$ (Lesniak et al. 1998; Marsavina \& Tomlinson, 2013), which for most applications is achieved at frequencies below $20 \mathrm{~Hz}$ (Lesniak et al., 1998). Such change is proportional to the first stress invariant range $\left(\Delta \sigma_{1}+\Delta \sigma_{2}\right)$ as stated by Marsavina \& Tomlinson, (2013) in the sample's surface, as shown in Eq. (1).

$$
\Delta \mathrm{T}=\frac{-\alpha_{T} \mathrm{~T}_{\mathrm{o}}}{\rho \mathrm{c}_{p}}\left(\Delta \sigma_{1}+\Delta \sigma_{2}\right),
$$

where $\alpha_{T}$ is the linear thermal expansion coefficient, $T_{0}$ is the reference temperature, $\rho$ is density, and $c_{p}$ is the specific heat under constant pressure. So, it seems convenient to place contact sensors on the surface, but often non-contact techniques are used to avoid touching components or when one need a whole-field measurement. This calibration method could be difficult to perform, due to the number of variables involved. Moreover, the radiation measured by an instrument includes emitted, transmitted, and reflected energy. However, with an appropriate calibration and with no presence of material non linearities or non-adiabatic factor, one can avoid the use of Eq. (1). For an isotropic material subjected to plane stress, Eq. (1) can be simplified to Eq. (2).

$$
S=K_{m}\left(\Delta \sigma_{1}+\Delta \sigma_{2}\right)
$$

where $S$ is the reading from the thermal camera and $K_{m}$ is the thermomechanical coefficient that can be either extracted from literature, calibrated in an experiment (Dulieu-Smith, 1995) or taken from a known sample's region that has known stress. According to Eq. (2), TSA gives the sum of the first stress invariant range when a body is subjected to alternating loads. Hence, post-processing needs to be done to separate the principal stress invariant range. 


\subsection{Photo-elasticity}

Photo-elasticity is perhaps one of the oldest methods for experimental stress analysis. For many years, it has been the preferred method for stress analysis because of its low cost and simplicity (Freire \& Voloshin, 2009; Masuda, 2004). Some parameters such as the stress concentration factor $K t$, or the stress intensity factor (SIF) were verified with the technique.

Light going through a birefringent material is used to determine the stress field in a sample subjected to a mechanical load. The material used has the capacity of changing its refractive properties when loaded. In other words, it exhibits temporary birefringence subjected to strain. This difference of refractive index acts as speed retardation for the polarized incident light, which is perceived as light of different colors (Freire \& Voloshin, 2009; Ramesh, 2000). The difference of principal stresses is a function of fringe number $N$, as given by Eq. (3).

$$
\sigma_{1}-\sigma_{2}=\frac{N}{t} \frac{E \lambda}{(1+v) K},
$$

where $t$ is the specimen thickness, $E$ is elastic modulus, $v$ is Poisson's ratio, $K$ is the strain optic coefficient of the material, and $\lambda$ is the used light source wavelength. The outer right terms in Eq. (3) can be simplified to the birefringent material's constant $f_{\sigma}$. Therefore, by observing the fringe order, one can establish the difference between principal stresses.

Photo-elasticity can be described using different mathematical models, such as the Poincare sphere, Jones calculus, Stoke's vector and quantum mechanics (Freire \& Voloshin, 2009). Nowadays, the technique is available in transmission and reflection mode (Masuda, 2004). Details about the color, fringe number, and bandwidth for the isochromatic bands formed in a birefringent material when subjected to a stress state are found in (Vishay, 2011). The technique used here assumed a linear elastic behavior, and mechanical properties of sample are independent of small changes in temperature.

\section{$2.3 \mathrm{DIC}$}

DIC started in 1982 at the University of South Carolina where a series of papers described a method to estimate deformation from a photographed deformed sample and how to compute rigid body rotation displacements (Sutton et al., 2009). DIC is a noncontact optical technique that uses one (DIC-2D) or more (DIC-3D) cameras to account for displacement, rigid body displacement, rigid body rotation, and out-of-plane displacement; the latter in case of DIC-3D (Vormwald et al., 2017). The technique uses a virtual mesh to discretize the area of interest (AOI) in smaller elements called subsets as depicted in Fig. 1a. Because of the nature of the problem dealt with, polar coordinates are used here using the stress notation presented in Fig. $1 \mathrm{~b}$.

DIC takes advantage of the grey intensity change within a subset in sequential photographs taken on a sample before and after deformation. Photographs are acquired digitally, i.e. by a digital camera (CMOS or CCD sensor), or by traditional methods which subsequently are digitized as pixel maps $f(x, y)$ making it possible to compare grey intensity before and after deformation to obtain displacement fields by using a minimization of a bits-correlation criteria, such as the squared sum of squared difference correlation criterion, SSD, as shown in Eq. (4). Other correlation criteria are normalized squared differences, zero-normalized squared difference, sum of absolute differences which have different usage depending on conditions (Castillo et al., 2020).
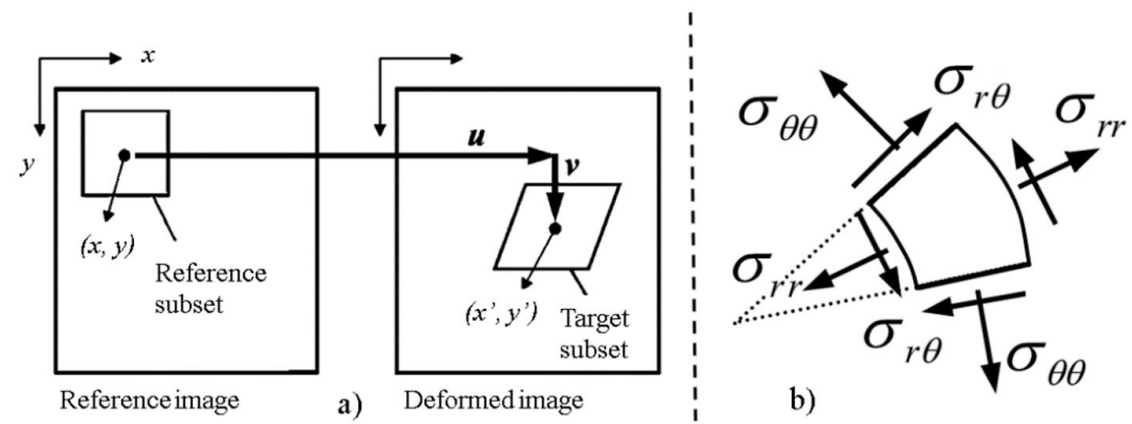

Fig. 1. a) Image before and after deformation for utilizing the DIC method; b) stress notation in polar coordinates coordinates

$$
S S D=\min \sum_{x=-M}^{M} \sum_{y=-N}^{N}\left[g\left(x^{\prime} y^{\prime}\right)-f(x, y)\right]^{2}
$$


where $f(x, y)$ and $g\left(x^{\prime}, y^{\prime}\right)$ represent the grey levels of reference before and after deformation; $(x, y)$ and $\left(x^{\prime}, y^{\prime}\right)$ are the coordinates of a point in the subset before and after deformation. Sutton et. al. (2009) warn that although the SSD is the least influenced by-lightvariation optimization criteria, it is also the most computationally expensive method. The coordinate ( $\left.x^{\prime}, y^{\prime}\right)$, after deformation, related to the coordinate $(\mathrm{x}, \mathrm{y})$, before deformation, using Taylor expansion shown in Eq. (5). Higher order terms can be mapped using iterative methods.

$$
\begin{aligned}
& x^{\prime}=x+u+\frac{\partial u}{\partial x} \Delta x+\frac{\partial u}{\partial y} \Delta y+\frac{1}{2} \frac{\partial^{2} u}{\partial x^{2}} \Delta x^{2}+\frac{1}{2} \frac{\partial^{2} u}{\partial y^{2}} \Delta y^{2}+\frac{1}{2} \frac{\partial^{2} u}{\partial x \partial y} \Delta x \Delta y \ldots \\
& y^{\prime}=y+\mathrm{v}+\frac{\partial \mathrm{v}}{\partial x} \Delta x+\frac{\partial \mathrm{v}}{\partial y} \Delta y+\frac{1}{2} \frac{\partial^{2} v}{\partial y^{2}} \Delta x^{2}+\frac{1}{2} \frac{\partial^{2} v}{\partial y^{2}} \Delta y^{2}+\frac{1}{2} \frac{\partial^{2} v}{\partial x \partial y} \Delta x \Delta y \ldots
\end{aligned}
$$

DIC provides independent values of displacement $u$, and $v$ in orthogonal directions, so there is no need for further postprocessing to use data (as opposed to photo-elasticity or TSA). The technique can be used to measure small or large samples, static or dynamics events, and rigid or soft materials. The speckles can be added by ink spray, can be etched, stamped, or even from natural patterns such as grain microstructure.

Once displacements are established using a correlation criterion, such as the one shown in Eq. (4), strains ( $\varepsilon$ ) can be calculated using the theory of elasticity Lagrangian relations as presented in Eq. (6), in polar coordinates, as presented in Fig. $1 \mathrm{~b}$.

$$
\varepsilon=\left[\begin{array}{cc}
\frac{\partial u_{r}}{\partial r} & \frac{1}{2 r}\left(\frac{\partial u_{r}}{\partial \theta}+\frac{2 \partial u_{\theta}}{\partial r}-\frac{u_{\theta}}{r}\right) \\
\frac{1}{2 r}\left(\frac{\partial u_{r}}{\partial \theta}+\frac{2 \partial u_{\theta}}{\partial r}-\frac{u_{\theta}}{r}\right) & \frac{1}{r}\left(\frac{\partial u_{\theta}}{\partial \theta}+u_{r}\right)
\end{array}\right]
$$

where $u$ is displacement in radial, $r$, or tangential, $\theta$, direction. By using elastic relations, strains and stresses are related as shown in Eq. (7) for plane stress.

$$
\left\{\begin{array}{l}
\sigma_{r r} \\
\sigma_{\theta \theta} \\
\sigma_{r \theta}
\end{array}\right\}=\frac{E}{1-v^{2}}\left[\begin{array}{ccc}
1 & v & 0 \\
v & 1 & 0 \\
0 & 0 & 1-v
\end{array}\right]\left\{\begin{array}{l}
\varepsilon_{r r} \\
\varepsilon_{\theta \theta} \\
\varepsilon_{r \theta}
\end{array}\right\}
$$

Extensive details about DIC can be found at (Sutton et al., 2009).

2.4 Stress Separation

Two ways to separate stress and another one link independent measurements are detailed here as follows.

\subsubsection{Independent stress measurement}

Dulieu-Smith (1995) reported TSA calibration directly from the experiment using two orthogonally placed SG in directions $i$ and $j$. Vishay Measurements recommends this method for photo-elasticity readings (Vishay, 2011). SG can be used to transform deformation readings into first stress invariant as shown in Eq. (8) for isotropic materials.

$$
\sigma_{i}+\sigma_{j}=\frac{E}{1-v}\left(\varepsilon_{i}+\varepsilon_{j}\right)
$$

then substituting Eq. (2) into Eq. (8), it will return the calibration constant as Eq. (9).

$$
K_{m}=\frac{E}{1-v} \frac{\left(\varepsilon_{i}+\varepsilon_{j}\right)}{S}
$$

It is noted that a second measurement is needed to use Eq. (9), and it must not interfere with TSA readings, i.e. SG should be allocated in the dark area of the specimen, making sure the wires neither shield nor reflect heat to the IR camera. 


\subsubsection{A known stress state}

Another way to extract the calibration constant is by performing a calibration trial against calculated stress. An area of the specimen or in a different specimen made out of the same material and tested under the same conditions, with a known analytic solution can be used to calculate known stress for the experiment. Then, reading $S$ from the camera is used in Eq. (2) to calculate $K_{m}$. Because the calibration constant $K_{m}$ may differ from point to point, the over deterministic least squares method (LSM) could be used to find the best fitting value.

\subsubsection{Assembling of independent readings}

As shown in Eq. (2) and Eq. (3), the TSA and photo-elasticity, respectively, do not provide independent readings of stress. Sakagami et al. (2004) and Murakami \& Yoshimura (1995) proposed a hybrid method combining TSA and photo-elasticity. The sum of the first stress invariant is constant as it is the difference of principal stresses. For the same measured point and when putting readings together, they form a linear system as shown in Eq. (10).

$$
\left[\begin{array}{cc}
\Delta \sigma_{1} & \Delta \sigma_{2} \\
\sigma_{1} & -\sigma_{2}
\end{array}\right]=\left\{\begin{array}{c}
C_{T S A} \\
C_{\text {Photoel }}
\end{array}\right\}
$$

Therefore, solving Eq. (10) for each measured point gives one separate values of principal stresses.

\subsection{Stress function}

If an exact description of stress fields is needed, a solution using an Airy stress function, $\phi(r, \theta)$, can be proposed. However, in order to be a stress function, it has to meet conditions (Sadd, 2014) as follows. The function has to be 2D biharmonic and scalar, and the fourth gradient for the proposed function has to be equal to zero. In the absence of temperature effects and body forces, Eq. (11) shows the governing biharmonic equilibrium condition for any Airy stress function in polar coordinates.

$$
\nabla^{4} \phi_{(r, \theta)}=\left[\frac{\partial^{2}}{\partial r^{2}}+\frac{1}{r} \frac{\partial}{\partial r}+\frac{1}{r^{2}} \frac{\partial^{2}}{\partial \theta^{2}}\right]^{2} \phi_{(r, \theta)}=0 \text {. }
$$

If $\phi(r, \theta)$ complies with Eq. (11), the stresses can be computed from the relations shown in Eq. (12). Furthermore, displacement fields $u_{r}$ and $u_{\theta}$ can be found by integrating strains from Eq. (6).

$$
\left[\sigma_{(r, \theta)}\right]=\left[\begin{array}{cc}
\frac{1}{r} \frac{\partial \phi}{\partial r}+\frac{1}{r^{2}} \frac{\partial^{2} \phi}{\partial \theta^{2}} & -\frac{\partial}{\partial r}\left(\frac{1}{r} \frac{\partial \phi}{\partial \theta}\right) \\
-\frac{\partial}{\partial r}\left(\frac{1}{r} \frac{\partial \phi}{\partial \theta}\right) & \frac{\partial^{2} \phi}{\partial r^{2}}
\end{array}\right]
$$

Finally, one needs to remember Michell general solution (Sadd, 2014), which is a generic function with terms that can be taken out at convenience. The solution complies with the conditions stated above to be an Airy stress function.

\section{Materials and methods}

A "keyhole" specimen of 1/4" thickness made of polycarbonate, Elastic modulus $E=2.6 \mathrm{GPa}$ and Poisson's ratio of $v$ $=0.37$ as shown in Fig. 2, was subjected to tensile loading. To have the sample free of residual stresses caused by the fabrication process, it was previously stress-relieved in an oven.

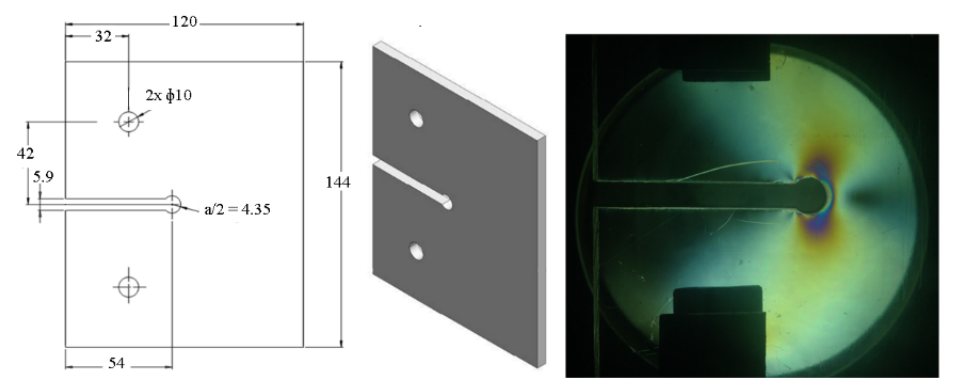

Fig. 2. Test coupon dimensions (in $\mathrm{mm}$ ), 3D view, and photo-elasticity set up. 
For TSA experiments, a FLIR A655sc micro-bolometer camera was used and the DeltaVision ® (Stress Photonics, Madison, WI) software was used to calibrate, to acquire and to process thermograms. DeltaVision ${ }^{\circledR}$ uses the least square method to adjust IR signals (Lesniak et al., 1998). A lock-in analyzer is employed to extract useful thermoelastic information from an exceedingly noisy output signal from the micro-bolometer. The lock-in analyzers used in TSA systems separate the measured signal into two orthogonal components: one in-phase and the other component at $90^{\circ}$ out-of-phase to the reference signal. This is done in such way because the sample's adiabatic mechanical response must be in-phase with the load frequency (Marsavina \& Tomlinson, 2013). A lock-in analyzer works by synchronizing the camera's thermal signal with either a signal from the load cell or a separate observation window within the same sample. In case heat is generated in regions with highstress gradients or if the loading frequency is not high enough for an adiabatic response, a phase change from the reference frequency occurs, which is visible as an out-of-phase signal (Dulieu-Smith, 1995; Marsavina \& Tomlinson, 2013). Moreover, conduction heat transfer is only a problem in TSA measurements when there are high-temperature gradients, i.e. a small component subjected to alternative stress and made out of a material with high thermal conduction (gold, silver, aluminum, copper, magnesium alloys, carbon, etc). Otherwise, heat loss by convection or significant radiation is negligible (DulieuSmith,1995). Extensive details on how DeltaVision ${ }^{\circledR}$ software works can be found in (Lesniak et al., 1998).

For photo-elasticity, a Vishay (Raleigh, NC) transmission polariscope, with quarter-wave-filters and equipped with a commercial fluorescent lightbox to achieve uniform white lighting, was used. It is important to use cold light as illumination, so the sample does not get heated which could affect measurements (Vishay, 2011). The material fringe, $f \sigma \mathrm{was} 7 \mathrm{~mm} / \mathrm{MPa}$. Photoelasticity patterns were digitally acquired with an iPhone $8 \mathrm{~s}{ }^{\circledR}$ camera (Apple inc, Palo Alto, CA). Pictures were taken both, in full color and with a monochromatic filter to avoid the problem that the combination between the light source and camera sensor may affect the quality of photo-elasticity images. Finally, it has to be said that although, digital image processing using RGB images and high-speed cameras are the norm for a modern photoelastic system, in this paper the fringe order estimation was manually performed.

For DIC measurements, the coupon was primed with white paint and sprayed painted with black ink to achieve the needed random speckles. Two, 5-MP CCD cameras (Point Grey GRAS-50S5M) equipped with 35-mm lenses, cameras were used to capture images and VIC Snap ${ }^{\circledR}$ was used for image acquisition, whereas 3D-VIC ${ }^{\circledR}$ (both from Correlated Solutions, Columbia NC) was used to process images. The mm to pixel resolution was 0.0697 .

At last, FEM 2D simulations were performed with ANSYS ® (Canonsburg, PA) using a linear elastic isotropic material model with triangular elements sizing of $2.75 \mathrm{~mm}$ in the bulk of the model. Around the keyhole outer edge and pin supports, $0.20 \mathrm{~mm}$ triangular elements in a $6 \mathrm{~mm}$ radius sphere were used. Plain stress conditions were assumed through the thickness.

Samples were subjected to tensile loads and readings were taken with the previously mentioned techniques. The goal was to obtain two sets of experimental measures (TSA and photo-elasticity in this case) to form the linear system as described by Eq. (8) at each measured point. One numerical result (FEM) was obtained to validate the separation method.

For DIC readings, the sample coupon was subjected to tensile forces with pauses for photographs at 52, 105, 160, 221, 270 and $307 \mathrm{~N}$ successively. The $3 \mathrm{D}-\mathrm{VIC}$ software readily delivers values for $\varepsilon_{\mathrm{xx}}, \varepsilon_{\mathrm{yy}}, \varepsilon_{\mathrm{xy}}, \varepsilon_{1}$ and $\varepsilon_{2}$. For TSA, the sample was subjected to alternating loads in the range of $460 \mathrm{~N}$ at 2 and $5 \mathrm{~Hz}$ loading rates. Calibration was done by making $\Delta \sigma_{2}=0$, from Eq. (1), in a second sample (see exemplary results in Fig. 3a) used exclusively for that reason. The $K_{m}$ constant was obtained using the procedure described in section 2.4.2. A comparison of a uniaxial known stress value from a dog bone sample made from identical material was done with IR camera readings, as seen in Fig. 3a. The FLIR ${ }^{\circledR}$ A655sc IR camera, with a $30 \mathrm{mK}$ sensitivity and spectral range from 7.5 to $14 \mu \mathrm{m}$, provided $S$ as a 640x480 matrix. To extract the $K_{m}$ constant several average values were taken. At last, for photo-elasticity, the sample was subjected to forces of $62,102,150,204$, and $252 \mathrm{~N}$.

\section{Results and discussion}

\subsection{TSA and photo-elasticity}

The highest $S$ value, as shown in Eq. (2), near the keyhole was looked for in order to find the $K_{t}$, as seen in Fig. 3 b. Once it was found, the data from a horizontal line (white dashed line in Fig. 3b) departing from the keyhole edge and in the opposite direction to the machined slit was processed using Eq. (2). Finally, to find the distance along pixels, a scale factor was extracted several times using a known dimension for the coupon. To verify TSA calibration, a FEM simulation was run using $E=2.4 \mathrm{GPa}, v=0.37$, and triangular elements. However, when comparing stress values obtained from different loads, it is recommended to normalize them by dividing the stress over the applied load. For photo-elasticity, there was a resolution problem at the edge due to residual stresses induced whilst machining the sample. In Fig. 4a through Fig. 4c the photoelastic fringes are shown for 62, 102, and $150 \mathrm{~N}$ in their full-color version. One can see how the fringes become blurry as one moves closer to the bore and such area appears to remain constant. It also can be observed how boundary conditions are filled at the slit borders as $\tau_{\text {nt }}$ is zero. Fig. $4 \mathrm{~d}$ and Fig. $4 \mathrm{e}$ shows the fringes for the sample loaded at 204 and $252 \mathrm{~N}$, respectively., which are shown in their monochromatic filtered version. 


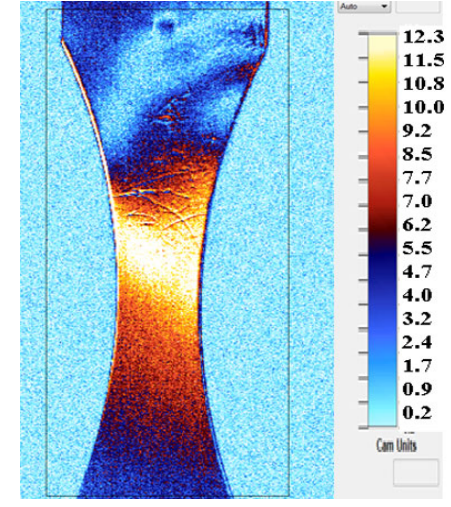

(a)

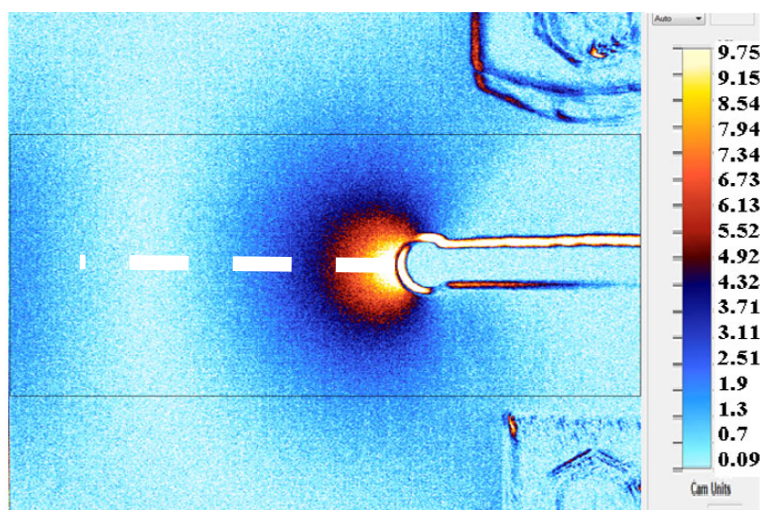

(b)

Fig. 3. Exemplary results of IR camera reading for $\Delta \mathrm{P}=460 \mathrm{~N}$ : a) calibration sample; b) keyhole sample

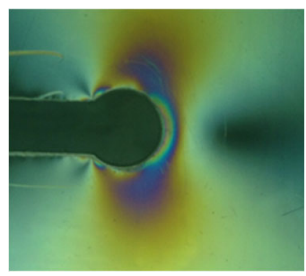

(a)

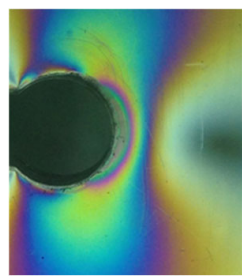

(b)

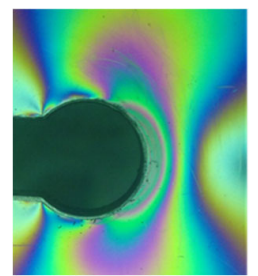

(c)

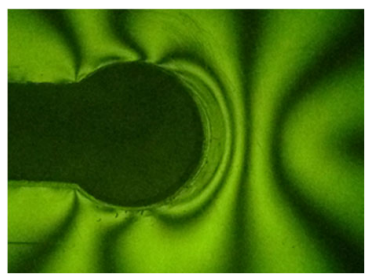

(d)

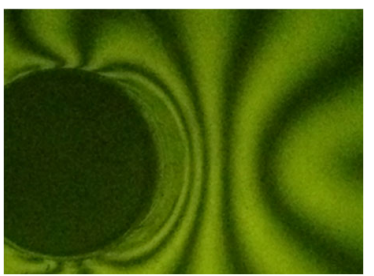

(e)

Fig. 4. Results of fringe bands at: a) $62 \mathrm{~N}$, b) $102 \mathrm{~N}$, c) $150 \mathrm{~N}$, d) $204 \mathrm{~N}$ and e) $252 \mathrm{~N}$

FEM and combined TSA + photo-elasticity results are shown in Fig. 5. (where the horizontal axis is the distance from the keyhole edge and the vertical axis is the normalized stress in $\mathrm{MPa} / \mathrm{N}$. Although there are seen slightly higher values for the experimental results, which will be discussed later, one can see an agreement between experimental and numerical results. Hence, the calibration method was validated. TSA data points were collected from the horizontal dashed white line depicted in Fig. $3 \mathrm{~b}$ and for photo-elasticity data, an analog white dotted line (see Fig. 3b) was used. The separation method was tested against FEM results, and it worked for stress values from the same load at the same point, at or away from the border.

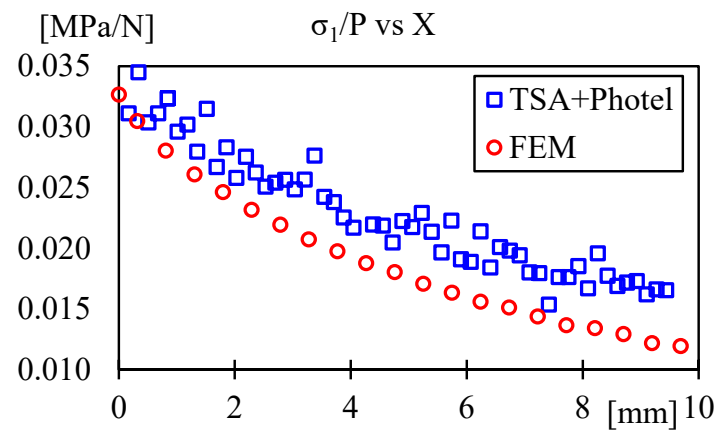

Fig. 5. Comparison of normalized first stress invariant along sample horizontal axis

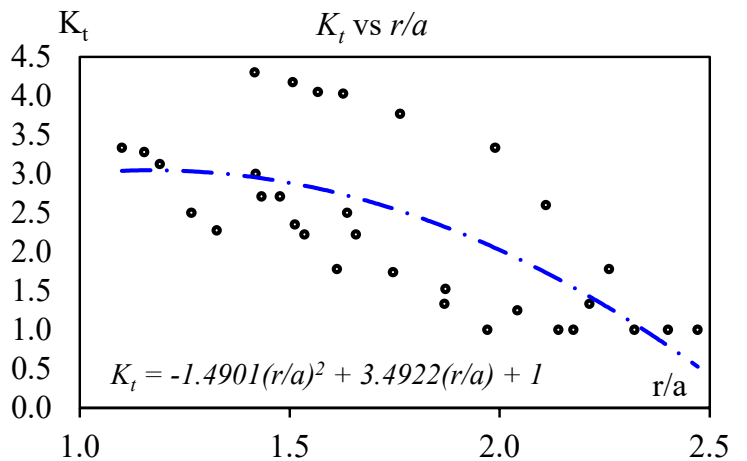

Fig. 5. $K_{t}$ vs $(r / a)$ from photoelasticity and TSA data

The stress concentration factor, $K_{t}$, can be calculated by Eq. (13).

$$
K_{t}=\frac{\sigma_{1}}{\sigma_{\text {Teor }}}
$$

Combined photo-elasticity and TSA results are presented in Fig. 5. It shows $K_{t}$ versus $r / a$, where $r$ is the distance from the center of the keyhole divided by the keyhole radius $a$. An order two polynomial interpolation was performed giving the relationship shown in Fig. 5, with the $K_{t}$ at the border $(r / a=1)$ being 3.02 . 


\subsection{DIC results}

As described before, DIC is able to provide orthogonal displacements measurements. Fig. 6a shows the parallel-to-load strain ( $\left.\varepsilon_{y y}\right)$ field as measured by DIC and Fig. $6 \mathrm{~b}$ shows the area of inspection (AOI) with a subset size (31 pixels) and step size ( 7 pixels) used for correlation analysis. It is observed how strains grow radially from outside and towards the keyhole center reaching a maximum at the outer most point of the notch, whereas they decay proportionally from the angular position reaching minimums at $\pm 90^{\circ}$.
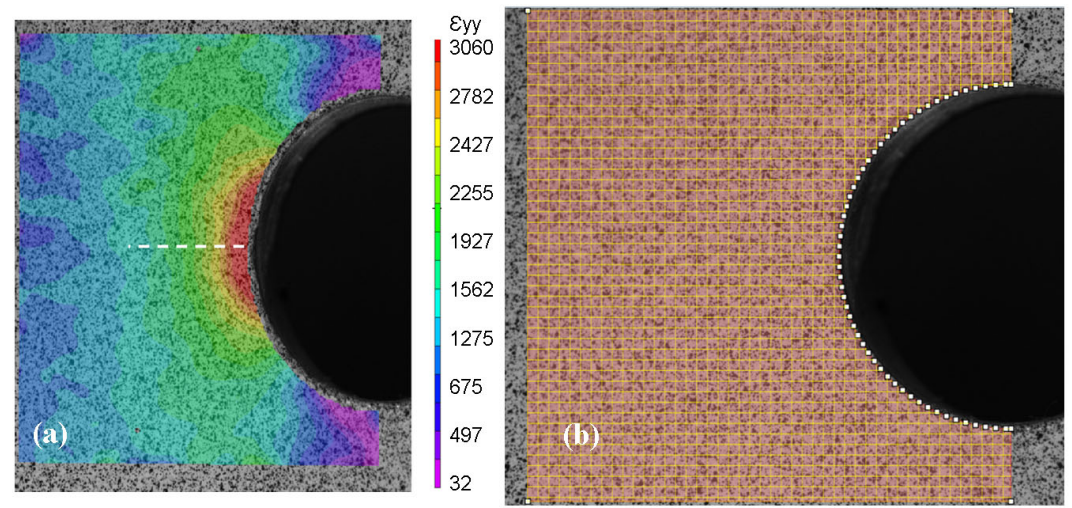

Fig. 6. a) DIC vertical strain, $\varepsilon_{y y}$, b) AOI with grid size used in the analysis.

Table 1 shows $K_{t}$ calculated with Eq. (14) for different acting loads from DIC data.

Table 1. Stress concentration factor, $K_{t}$, obtained with DIC data

\begin{tabular}{cccccccc}
\hline$\sigma_{\mathrm{y} \text { DIC }}(\mathrm{MPa})$ & 0.89 & 1.78 & 2.67 & 3.55 & 4.45 & 5.33 \\
\hline $\mathrm{K}_{\mathrm{t}}$ & 2.94 & 2.83 & 2.88 & 2.94 & 2.88 & 2.94 \\
\hline
\end{tabular}

which gives an average $\mathrm{K}_{\mathrm{t}}$ of 2.91 , very close to results presented in Fig. 5 ( $K_{t}$ from combined photo-elasticity and TSA). Results from a FEM simulation under $251 \mathrm{~N}$ for the perpendicular-to-load strain are shown in Fig. 7a, whereas results for the parallel-to-load strain are shown in Fig. 7b. The perpendicular -to-load strain shows the characteristic butterfly-like field in front of a notch.

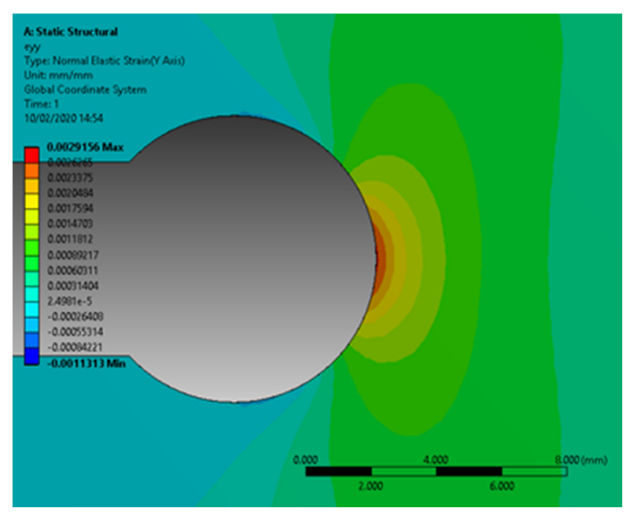

(a)

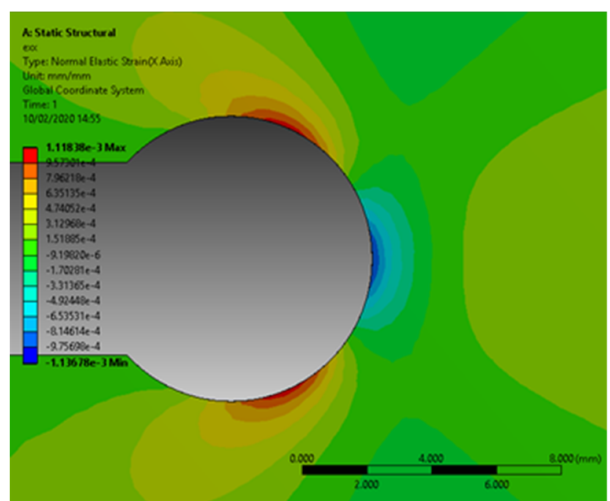

(b)

Fig. 7. FEM normal strain for the $251 \mathrm{~N}$ load, a) perpendicular-to-load, b) parallel-to-load

Fig. 9 shows a comparison between normalized stresses as follows: TSA \& photo-elasticity obtained through matrix Eq. (8), labeled $\sigma_{1}$ and $\sigma_{2}$, DIC labeled $\sigma_{1}$ DIC and $\sigma_{2}$ DIC, and FEM results labeled $\sigma_{1}$ FEM and $\sigma_{\mathrm{s}}$ FEM. It can be seen an agreement between TSA \& photo-elasticity with FEM for both directions. However, for DIC normal stress $\left(\sigma_{1}\right.$ DIC) the tendency agrees with other methods, but values are a little lower than their counterpart. $\sigma_{1}$ FEM shows values a little higher when getting close to the edge than both experimental values. This may be explained by the traction residual stress product of the coupon's fabrication process that the linear elastic numerical simulation cannot reproduce. 


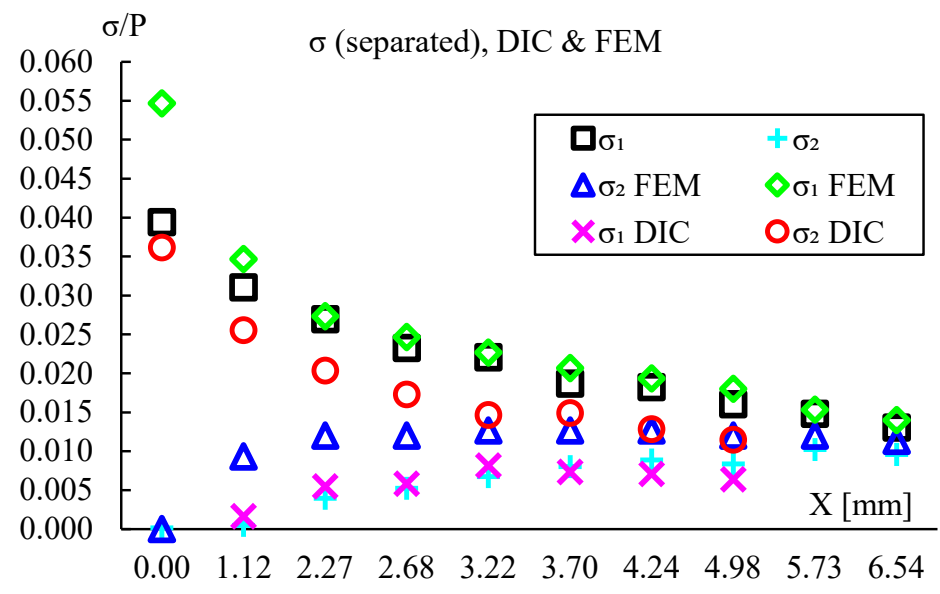

Fig. 8. Normalized separated stresses obtained with TSA \& photoelasticity, DIC and FEM stress vs distance from the keyhole border

\subsection{Results with the Airy stress function}

Michell's general solution includes some $r^{2}$ terms. So as $r$ gets larger, the stresses would become infinite, which in this situation clearly, it is not the case. Thus, the $r^{2}$ terms were left out. The other boundary condition for this problem is for $\sigma_{r \theta}(a, \theta)=0$. Therefore, following these previous observations and recommendations from Sadd (2014), an Airy stress's function is proposed, as shown in Eq. (14).

$$
\phi_{(r, \theta)}=b r \ln (r) \cos \left(\frac{\theta}{2}\right) ; r>a
$$

The stresses are found using Eq. (6), which produced Eq. (15).

$$
\left.\begin{array}{l}
\sigma_{r r}=b r^{-1}[0.75 \ln (r)+1] \cos \left(\frac{\theta}{2}\right) \\
\sigma_{\theta \theta}=b r^{-1} \cos \left(\frac{\theta}{2}\right) \\
\sigma_{r \theta}=0.5 b r^{-1} \sin \left(\frac{\theta}{2}\right)
\end{array}\right\} ; r>a
$$

Eq. (15) are consequent with the boundary conditions exhibited by the experiments. The constant $b$ can be guessed until it fits boundary conditions, or it can be data fitted to experimental data. Assuming linear elastic conditions, the von Misses stress (the chosen failure criterion) is calculated for the stresses in Eq. (15) and also for the measured DIC strain fields. The stress values from the Airy stress function, Eq. (15), were converted to strains using the relations shown in Eq. (7). Then, linear data fitting is performed as shown in Eq. (16) for the available measured $i$ points.

$$
e r r_{i}=\frac{\mathcal{E}_{D I C}-\mathcal{E}_{\text {Airy }(b, r, \theta)}}{\mathcal{E}_{\text {DIC MAX }}}
$$

where err is the difference to be minimized, $\varepsilon_{\text {DIC }}$ is the measured strain field, $\varepsilon_{\text {DIC MAX }}$ is the maximum strain value at the keyhole, $\varepsilon_{\text {Airy }}$ is the proposed strain field in polar coordinates $(\mathrm{r}, \theta)$, so $b$ is the parameter that minimizes err. One can see that if more than one point is used, Eq. (16) yields an over-determined system, which can be solved by the least squares method. The obtained constant $b$ was $7.45 \mathrm{MPa} / \mathrm{mm}$. Fig. 9 shows the comparison of experimental and theoretical first principal strain fields with Eq. (16). There are regions of about $40 \%$ error but those are located at the bottom right corner, probably due to a low DIC correlation value. All in all, in front of the keyhole, where a potential crack could emerge and grow, there is a low error. 


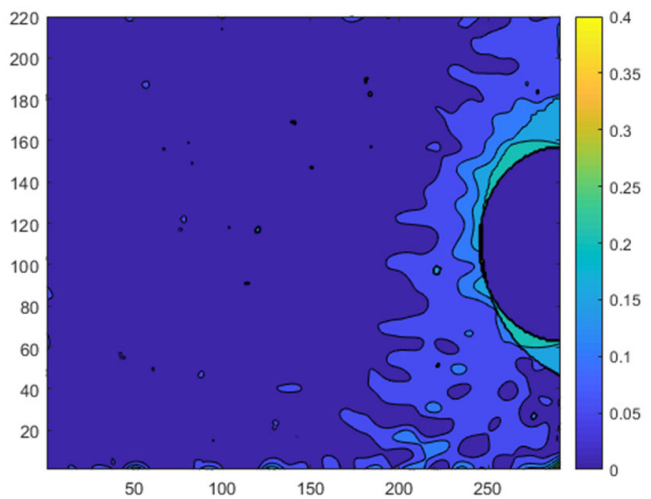

Fig. 9. error in principal strain between DIC and Airy methods

Fig. 10 shows the comparison of the first principal strain obtained with DIC with the proposed stress function. One can see the similitude in shape and values between the two methods, and also with results from FEA as seen in Fig. 7 for parallelto-load strain. The combination loading - geometry makes, in both cases for the DIC measured field and the Airy field, how the first principal strain decays radially departing from the edge.
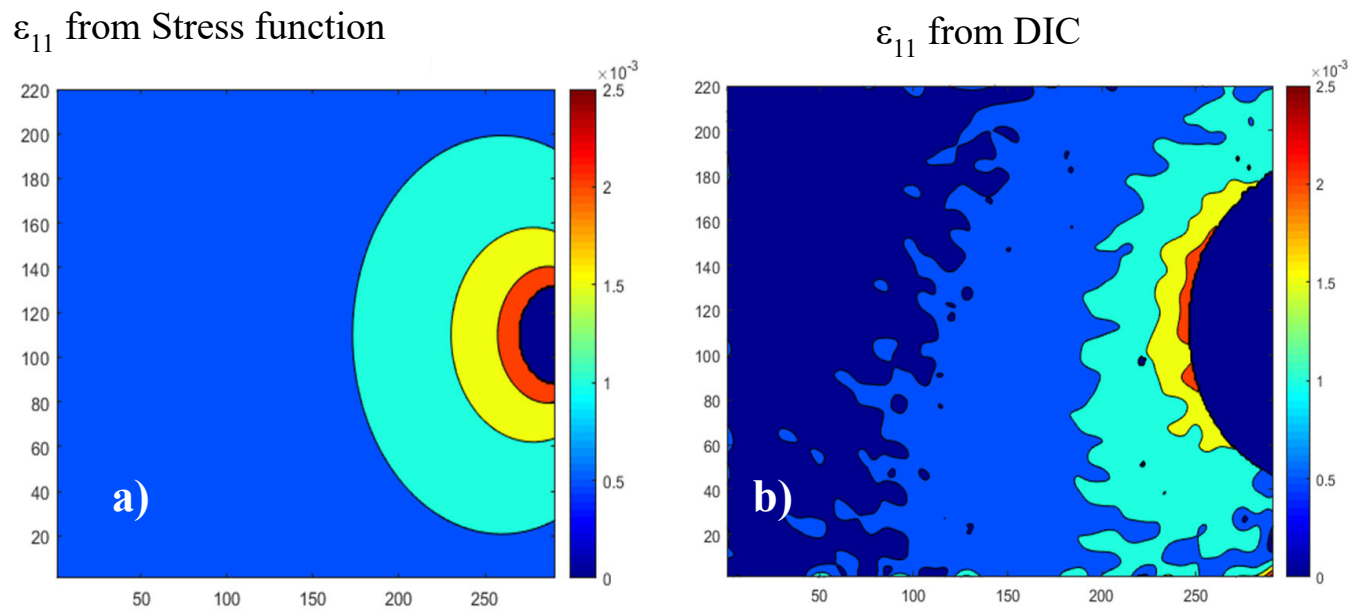

Fig. 10. First principal strain obtained with a) stress function, b) DIC.

The obtained $b$ constant was tested with the shear stress, $\sigma_{\mathrm{r} \theta}$ for the Airy stress function and compared to the DIC measured field, $\varepsilon_{\mathrm{r} \theta}$, as shown in Fig. 11. One has to remember that in this case shear stress $\sigma_{\mathrm{r} \theta}$ is null at the border, $r=a$ and $\theta=0$ which is certain despite the experimental error from the DIC technique.
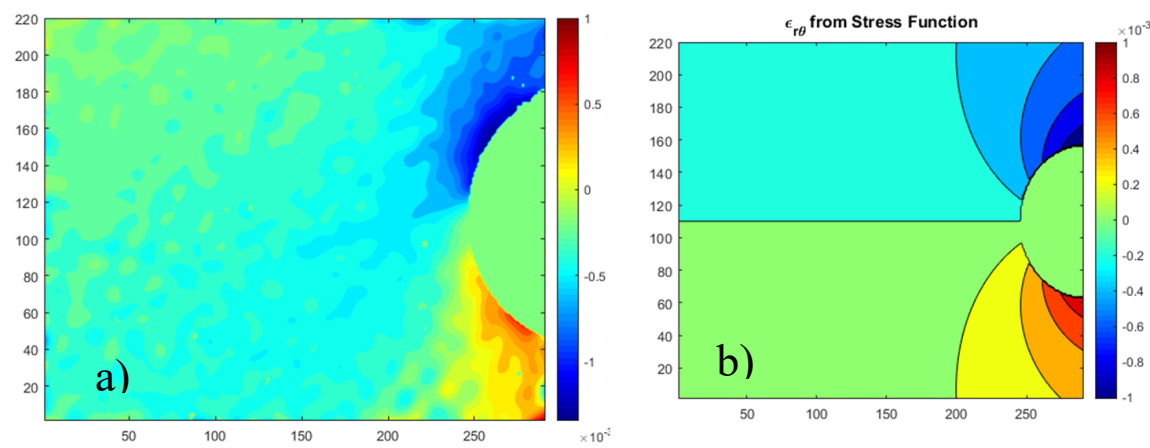

Fig. 11. Shear strain, $(r, \theta)$, obtained with a) DIC and b) stress function 


\section{Conclusion}

Photo-elasticity and TSA are techniques that measure stress directly as opposed to strain gages or DIC which measure change in electrical resistance or displacements, respectively. Photo-elasticity and TSA however, do not provide independent stress measurements. Therefore, they must be separated so useful information can be extracted and properly analyzed. The evaluated stress separation technique was successful telling apart experimental stresses from TSA and photoelasticity when applied to a polycarbonate keyhole sample under tension. Results from such separation were compared with results from the DIC technique and FEA. The calculated stress values lead to a very close stress concentration factor, $K_{\mathrm{t}}$ used as a benchmark. Furthermore, all of the experimental techniques used here are capable of only obtaining superficial readings, so that the method can be applied to isotropic materials under plane stress conditions. Careful results interpretation should be done in case the method is applied to a sample subjected to plane strain because most likely, the superficial stresses found with the techniques are not going to represent the maximum state of stress.

Although the IR equipment used in Dulieu-Smith (1995) was very different to what is available today for TSA, a direct calibration from experiment properties, as shown in Eq. (2) was done here. Dulieu-Smith (1995) compared both methods, the one presented in Eq. (2) seems to be the most complicated method and prone to error due to uncertainties of material properties. A much simpler approach was taken here by comparing IR camera readings to a known stress state.

It was observed that the values of $\sigma_{\mathrm{xx}}$ and $\sigma_{2}$ as well as $\sigma_{\mathrm{yy}}$ and $\sigma_{1}$ are almost identical in DIC, which is expected in a uniaxial loading case. The difference may be due to a lack of alignment between the sample's holes and loading device axis and to the residual stress product of the fabrication process. Additionally, DIC appeared to provide slightly lower values than other techniques. This could be due to the correlation parameters used to run the correlation (subset, step, and Lagrangian filter) which can smooth data for large setting values. Optimal combination of parameters, along with average speckle size, is an ongoing topic of research. Another source of difference could be attributed to the fact that the sample endured local plastic deformation due to the rapid alternating stress used when testing with TSA.

The method to extrapolate the stress concentration factor seems valid as seen in Fig. 6 and Fig. 8. Depending on the technique used, there is an inherent measuring error on the edge that prevents one from calculating stresses at such borders. By measuring the appropriate variables and calculating stresses along a horizontal line, one can extrapolate the actual value of $K_{\mathrm{t}}$.

A stress function was proposed, validated, and experimentally tested, finding it represents well the stress fields when compared to experimentally acquired DIC and FEM simulated strain fields with a polar coordinate system located in the center of the keyhole. Furthermore, the function describes the close-to-edge stress field, but only for the opposite-to-the slit side of the sample. Finally, the stress function leaves out of the stress description the support pin areas which are expected not to be the areas with the highest stress concentration factor.

\section{References}

Allison, I. M. (1998). A rapid method for stress separation in two dimensional photoelastic models. Strain, 35(3), 83-86.

Anthony, N., Cadenazzi, G., Kirkwood, H., Huwald, E., Nugent, K., \& Abbey, B. (2016). A Direct Approach to In-Plane Stress Separation using Photoelastic Ptychography. Scientific Reports, 6.

Castillo, I. M., Peñaranda, A. E., \& Diaz, J. G. (2020). Implementación de la Técnica de Correlación de Imágenes Digitales con Software Libre. Aibi, 8(3), 25-32.

Cavaco, M. A. M., R.Viotti, M., \& Albertazzi Jr, G. A. (2018). Pipeline Stress Analysis Through Stress Function Fittings. Latin American Journal of Solids and Structures, 15(9).

Dulieu-Smith, J. M. (1995). Alternative calibration techniques for quantitative thermoelastic stress analysis. Strain, 31(1), 916.

Freire, J. L., \& Voloshin, A. (2009). PHOTOELASTICITY. In Encyclopedia of Life Support Systems (EOLSS) (1st ed.). Oxford ,UK: UNESCO. Retrieved from http://www.eolss.net/outlinecomponents/Experimental-Mechanics.aspx

Jobin, T. M., Khaderi, S. N., \& Ramji, M. (2020). Experimental evaluation of the strain intensity factor at the rigid line inclusion tip embedded in an epoxy matrix using digital image correlation. Theoretical and Applied Fracture Mechanics, $106,102425$.

Lesniak, J. R., Boyce, B. R., \& Howenwate, G. (1998). Thermoelastic Measurement Under Random Loading. In Spring SEM conference. Houston, TX: SEM. https://doi.org/10.1111/j.1747-1567.1998.tb02410.x

Marsavina, L., \& Tomlinson, R. A. (2013). A review of using thermoelasticity for structural integrity assessment. Frattura Ed Integrità Strutturale, 8(27), 13-20.

Masuda, Y. (2004). The Past and Present or a History of Photoelasticity and its Future Image of Becoming Automated. Journal of the Japanese Society for Experimental Mechanics, 4(1), 3-10.

Molteno, M. R., \& Becker, T. H. (2015). Mode I - III Decomposition of the J -integral from DIC Displacement Data. Strain, 51(6), 492-503.

Murakami, Y., \& Yoshimura, M. (1995). Development of System Resolving All Stress Components in Thermoelastic Stress 
Analysis. JSME International Journal Series A, 61, 2482-2488.

Niedenfuhr, W. F. (1957). On Choosing Stress Functions in Rectangular Coordinates. Journal of the Aeronautical Sciences, 24(6), 456-472.

Ramesh, K. (2000). Stress Separation Techniques. In K. Ramesh (Ed.), Digital Photoelasticity (pp. 303-346). Berlin: Springer Verlag.

Rao Kavati, D., \& Nomura, S. (2006). Airy stress function for two dimensional inclusion problems. In ASME International Mechanical Engineering Congress and Exposition, IMECE. Chicago.

Sadd, M. H. (2014). Elasticity. (Elsevier, Ed.) (3rd ed.). Kingston, Rhode Island: Elsevier.

Sakagami, T., Kubo, S., Fujinami, Y., \& Kojima, Y. (2004). Experimental Stress Separation Technique Using Thermoelasticity and Photoelasticity and Its Application to Fracture Mechanics. JSME International Journal Series A, 47(3), 298-304.

Selvadurai, A. P. (2000). The biharmonic equation. In Partial Differential Equations in Mechanics 2. Berlin: Springer.

Solarguen, M., Alegre Calderón, J., Bravo Díez, P. M., \& Cuesta Segura, I. (2009). Stress-separation techniques in photoelasticity: A review. The Journal of Strain Analysis for Engineering Design, 45(1), 1-17.

Sutton, M. A., Orteu, J. J., \& Schreier, H. (2009). Image Correlation for Shape, Motion and Deformation Measurements: Basic Concepts, Theory and Applications. Boston: Springer.

Tabanyukhova, M. (2019). Photoelastic Analysis of the Stressed State of a Flat Element with Geometrical Stress Concentrators (Cutout and Cuts). Key Engineering Materials, 827, 330-335.

Triana-Infante, C. A., Varón-Durán, M., \& Pastor-Abellán, D. (2014). Validación de sensores basados en redes de difracción de bragg (FBGS) para deformación y temperatura. ITECKNE, 2, 172-182.

Vishay. (2011). Principal Stress Separation in PhotoStress ${ }^{\circledR}$ Measurements. Document Number: 11217. USA.

Vormwald, M., Hos, Y., Freire, J. L. F., Gonzáles, G. L. G., \& Díaz, J. G. (2017). Variable mode-mixity during fatigue cycles - crack tip parameters determined from displacement fields measured by digital image correlation. Frattura Ed Integrità Strutturale, 11(41), 314-322.

Yoneyama, S., Morimoto, Y., \& Kawamura, M. (2005). Two-dimensional stress separation using phase-stepping interferometric photoelasticity. Measurement Science and Technology, 16.

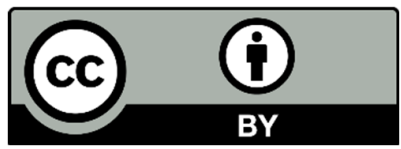

(C) 2022 by the authors; licensee Growing Science, Canada. This is an open access article distributed under the terms and conditions of the Creative Commons Attribution (CC-BY) license (http://creativecommons.org/licenses/by/4.0/). 